\title{
Real time control and monitoring of grid power systems using cloud computing
}

\author{
Nachiket Kulkarni ${ }^{1}$, S. V. N. L. Lalitha ${ }^{2}$, Sanjay A. Deokar ${ }^{3}$ \\ ${ }^{1}$ Koneru Lakshmaiah Education Foundation, Vaddeswaram, Guntur, Andhra Pradesh, India \\ ${ }^{2}$ Electrical \& Electronics Engineering Department, Koneru Lakshmaiah Education Foundation, India
}

\begin{abstract}
Article Info
Article history:

Received May 17, 2018

Revised Oct 3, 2018

Accepted Nov 2, 2018

Keywords:

Cloud computing

Energy management

Grid system

HMI

PLC

Real time monitoring

Smart grid

ABSTRACT

The use of grid power systems based on the combinations of various electrical networks, information technology, and communication layers called as Smart Grid systems. The technique of smart grid suppressed the problems faced by conventional grid systems such as inefficient energy management, improper control actions, grid faults, human errors, etc. The recent research on smart grid provides the approach for the real-time control and monitoring of grid power systems based on bidirectional communications. However, the smart grid is yet to improve regarding efficiency, energy management, reliability, and cost-effectiveness by considering its real-time implementation. In this paper, we present the realtime design of efficient monitoring and control of grid power system using the remote cloud server. We utilized the remote cloud server to fetch, monitor and control the real-time power system data to improve the universal control and response time. The proper hardware panel designed and fabricated to establish the connection with the grid as well as remote cloud users. The authenticated cloud users are provisioned to access and control the grid power system from anywhere securely. For the user authentication, we proposed the novel approach to secure the complete smart grid system. Finally, we demonstrated the effectiveness of real-time monitoring and control of the grid power method with the use of structure of practical framework.
\end{abstract}

Copyright $(2019$ Institute of Advanced Engineering and Science. All rights reserved.

\section{Corresponding Author:Nachiket kulkarni}

Nachiket Kulkarni,

Koneru Lakshmaiah Education Foundation,

Vaddeswaram, Guntur, Andhra Pradesh, India - 522502.

Email: knachiket62@gmail.com

\section{INTRODUCTION}

Nowadays, there is significant research and development is carried out on the smart grid with the aim of improving the effective energy management, efficient power control and monitoring. The smart grid is known as various networks of electrical, communication systems as well as Information \& the Technology of Communication (ICT) standards. The smart grid provides the power control more effective, reliable and efficient monitoring using the emerging advancement in ICT [1]. The smart grid helps to monitor the use of power and match the power consumption with the system load. The remotely controlled smart grid delivered the self-monitoring, bidirectional communications, self-controlling, and self-healing communication for transmission, production of energy, control of energy, distribution of energy, and power observation to balance the demand and supply [2]. As compared to traditional grid systems, it is required to manage the number of smart grid in reliable, efficient, scalable and secure approach. The smart grid system should extend their communication networks and control all the data over the remote distributed data storage. Therefore, to improve the smart grid system performance, recently the number of studies reported the elements of cloud computing into the smart grid. The cloud computing is emerging technology to remotely 
store, monitor and control any data from any place in the world. The cloud computing frameworks are playing the significant role in the framework of next smart grid method. For smart grid systems, cloud computing helps to access, monitor and control the real-time smart grid data remotely from any place and anytime through the appropriate communication interfaces [3], [4]. More, here we presented the key factors with the utilization of cloud computing into the grid automation method.

The substation system is consisting of various devices; the controlling of such devices can be done remotely from the substation data centres. The automation of substation plays the significant role in monitoring and control of power system. Such systems address the numbers of challenges. Flexibility of the substation and power system has been achieved by efficient real time controlling of substation remotely. However, the grid system automation leads to the various problems during its operation which will change the typical behaviour of electrical devices in power system. Therefore, computing facilities are one of the essential challenges in grid system automation [5]. Traditionally, the electric grid system based on their database servers and storage devices, but for communication with the substation which situated at the remote location, the distributed network architecture is required. Cloud computing technique can provide this network. In power grid systems, the substation plays the significant role as there is the enormous amount of data generated, enticing the need of massive data storage facilities [6].

In this paper, our main objective is to design real-time monitoring and control of substation power data remotely in the efficient, secure and effective manner using the real-time cloud computing framework from any place and any time. As per the report of Indian Grid failure [7], [8], the leading cause of cascade tripping was the over-drawing of power between eastern and northern grid. Also in the report, there has been stated the requirement for the grid security against such over-drawl conditions. This research shows the method which has been utilized to observe the status of grid parameters like load, VA, voltages, and current. This system aims to acquire the grid parameters remotely and then control them to prevent the over drawling conditions, although it is the initial step towards the future smart grid systems.

This research presented as, Section 2, the review on application of smart grids cloud computing is described. In Section 3, the methodology and designs presented for efficient real-time monitoring of grid power systems using cloud computing. Section 4 shows the detailed design of hardware panel of Real-time Control and Monitoring (RTCM) Device. In Section 5, the results and discussions are presented. Finally, Section 6 gives the conclusion, and future directions based on this research work are presented.

\section{RELATED WORK}

In grid systems, the process of energy management has been known as energy conservation, controlling \& observations. The management of power is main research problem into smart grid. This part shows the study on conventional grid systems and cloud-based recent works for smart grid.

Further, Ming Chen et al. [8] dismembered the need and feasibility of cloud figuring advancement in power dispatching and displayed the Deployment Method of power dispatching computerization system based on cloud handling. Ordinary systematization of power dispatching technique; fast movement of cutting-edge limits; and huge change unwavering quality of IT frameworks can be accomplished utilizing cloud figuring innovation. It diminishes authoritative expenses, and it tackles the inconsistency between progressive management and "coordinated development" in the energy segment.

In [9], scientist proposed cloud dispatching, a kind of cloud enrolling based general system of adroit dispatching centres. The cloud handling based layered plan consolidates (1) Physical Resource Layerphysical hardware, arrange resources and application systems, for instance, SCADA, EMS, TMR, WARMS, (2) Virtual Resource Layer - depict resources in various sorts into virtual resources, (3) Cloud Service layer groups virtual resources into services, that are introduced on the clouds, (4) Cloud Management Layer - gives facilitated management of cloud services for customers, and (5) Cloud Access Layer - the way customer access cloud dispatching. It joins the present resources demands among various dispatching centres, reduces the system improvement and expansion cost and upgrades general dispatching business limit.

Yang et al. [10] prescribed that cloud stages are proper to help immense data and computationallyheightened, constantly on applications. The Cloud Access Layer keen network structure needs to pass on all around. To change the constant demand and supply twists, fast coordination and examination of data that streams from different savvy meters at the same time are required that requires the adaptable programming stage. Thus in such conditions, cloud stages fill in as fundamental parts due to the distinctive favourable circumstances they offer.

In [11], author Rajeev and Ashok proposed a structure for planning cloud enlisting applications for micro-grid management as different modules, for instance, system, power management, and service. The structure and power management modules are used for undertaking arranging and micro-grid power management independently. The differing chairmen circulate their service depiction using the service 
module. With the use of cloud preparing, the external figuring devices can facilitate with the internal ones. Thusly, the amount of reinforced customers increases as proposed by Rajeev and Ashok. In such a way, joining virtual energy sources with the present energy stockpiling devices and the energy exchange segment can be refined among the micro-grids to meet the energy necessities from purchasers.

In [12], Vazguez et al. expected to give the capacity to manufacture discretionary complex network frameworks ready to maintain the request required by any given administration, exploiting the compensation per-utilize display and the apparently boundless limit of the distributed computing worldview.

In [13], Moslehi et al. explored the unwavering quality effects of real brilliant lattice assets, for example, sustainable, request reaction, and capacity. They watched that a perfect blend of these assets prompts a compliment net request that in the long run complements unwavering quality difficulties further.

In [14], Tooth et al. demonstrated that cloud movements, including cloud machine, dispersed capacity, disseminated registering and cloud security, acknowledge a basic part in redesigning the cataclysm status level and the versatility of energy structures to catastrophes. Moreover, by utilizing cloud advancements, the level of energy structure streamlining and the exactness of energy system beguilement can expand.

In [15], Fang et al. proposed a redesign issue of utilizing the cloud an area to lessen the cost of data organization in the SG. They displayed a cloud-based SG data organization demonstrate and presented a cloud and system resource streamlining structure to manage the cost diminishment issue in cloud-based SG data stockpiling and figuring.

\section{METHODOLOGY}

To alleviate traditional smart grid systems problems, automation is the best solution as discussed earlier. This section discussed about overall framework \& algorithms for given proposed method for enhancing the improved smart grid performance. We designed the system to collect the real-time power system data under the operating conditions using the cloud computing framework remotely. We believe that fetching every minute electrical data can help to prevent the significant blackouts. Using the cloud computing technology, the critical conditions can be prevented by remote operations such as data processing, analysis, and immediate monitoring. Figure 1 shows the working of proposed methodology of this paper. We introduced a novel hardware panel design which is used to monitor the status of grid parameters like load, VA, voltages and current effectively. The hardware device further interfaced with the cloud storage to access the actual minute to minute electrical data from any place and anywhere by the authenticated users.

\section{Authenticated users}

\section{Cloud storage devices \&Global data}

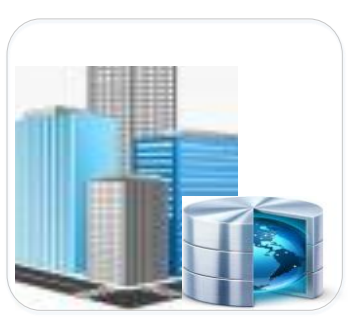

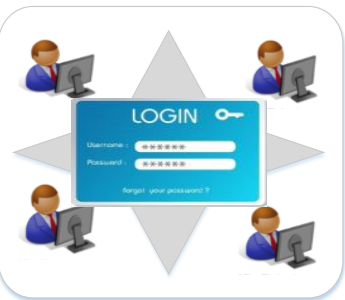

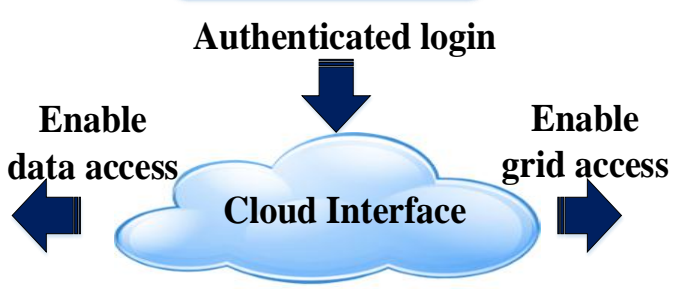

Grid automation

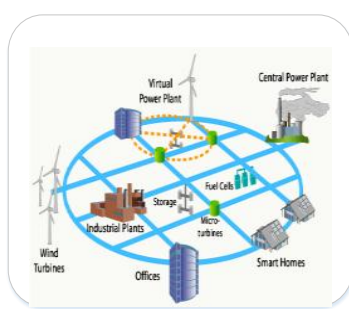

Figure 1. Process of the proposed work

As for showing in Figure 1, the authenticated user login helps to prevent any illegal activities in the network. We designed the hardware panel in this paper which acts as the interface between cloud services and the grid systems. Therefore designing of hardware panel is the important goal of this paper. Incoming sections, we elaborate the design and methods used in this paper. 


\subsection{Design of hardware panel}

The goal of this research is to implement the hardware panel to establish the interface between the remote authenticated cloud users and power grid systems to access, monitor and control the electrical grid data anytime and anywhere. Figure 2 is showing the proposed single line diagram for the hardware panel in which the two generators feed the grid. Further, these two generators are feds by it's their local loads. The algorithm of a project is used to isolate the grid loads during the situations like over-drawl, whereas local loads continue with the power supply.

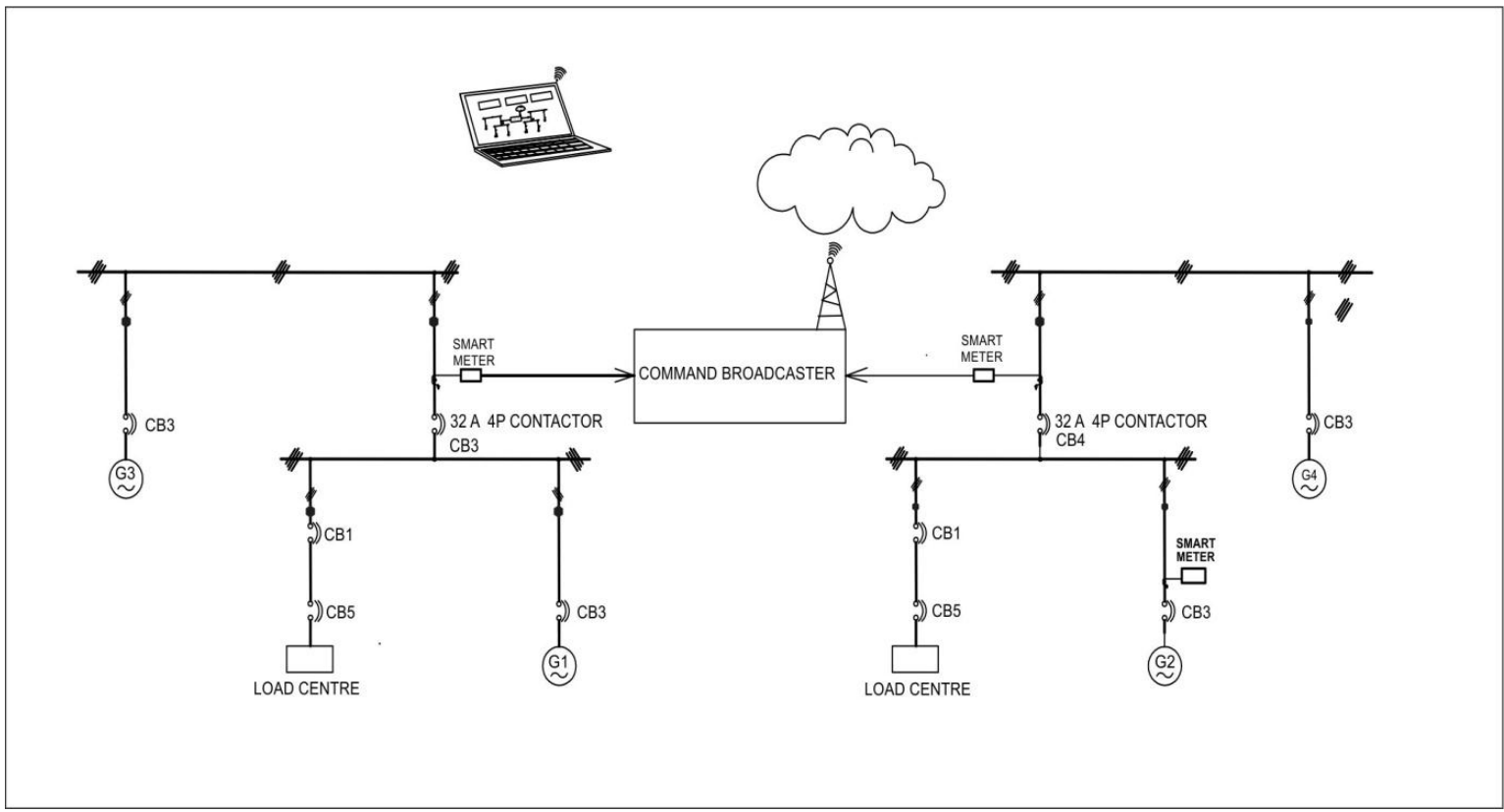

Figure 2. Real time control and monitoring of power using cloud computing

The load manager senses the parameters of the grid such as voltage, current, KW, and KVA. The load managers interfaced with the PLC (programmable logic controller), and PLC then interfaced with Human Machine Interface (HMI) system. The HMI system is further uploading the current electrical data on the cloud to access data from the remote systems using PC or Laptop. Access to the device can be achieved through internet connected systems (PC/Laptop) having authenticated login id and password. With this log in the user can edit/change the set/reference values in the program in PLC-CPU. Figure 3 shows the above process flow by considering the two generators and load

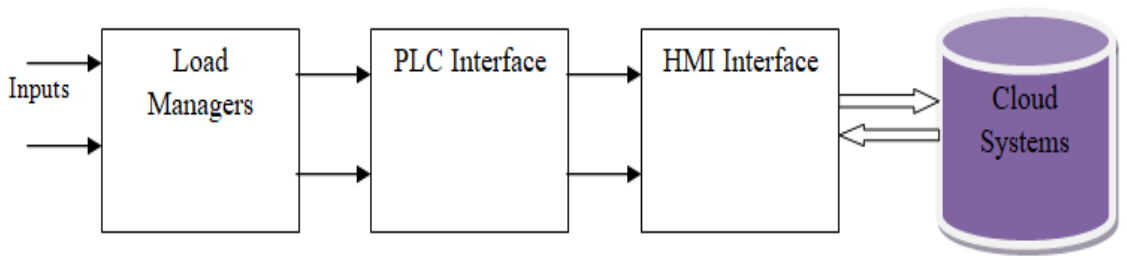

Figure 3. Overall processing flow of proposed architecture

\subsection{Authenticated cloud interface}

To provide the security to grid data misuse, we designed the user authentication algorithm for each user to access the electrical data through the cloud interface. The end clients need to finish their verification procedure required by the service supplier at whatever point they utilize cloud services. A client registers with offering individual data, and a service supplier gives a client's distinguishing proof and a validation technique for client confirmation after enrolment is finished. At that point the client utilizes the ID and the 
confirmation technique to work the client verification when the client accesses to utilize a cloud service. The process of user authentication is elaborated through three algorithms below for each step such as user registration, login, and authentication respectively. To start the registration process, the user has to provide the personal identification details to the server. The server offers a unique token to the user by processing the features provided by the user. The steps involved in user registration process given:

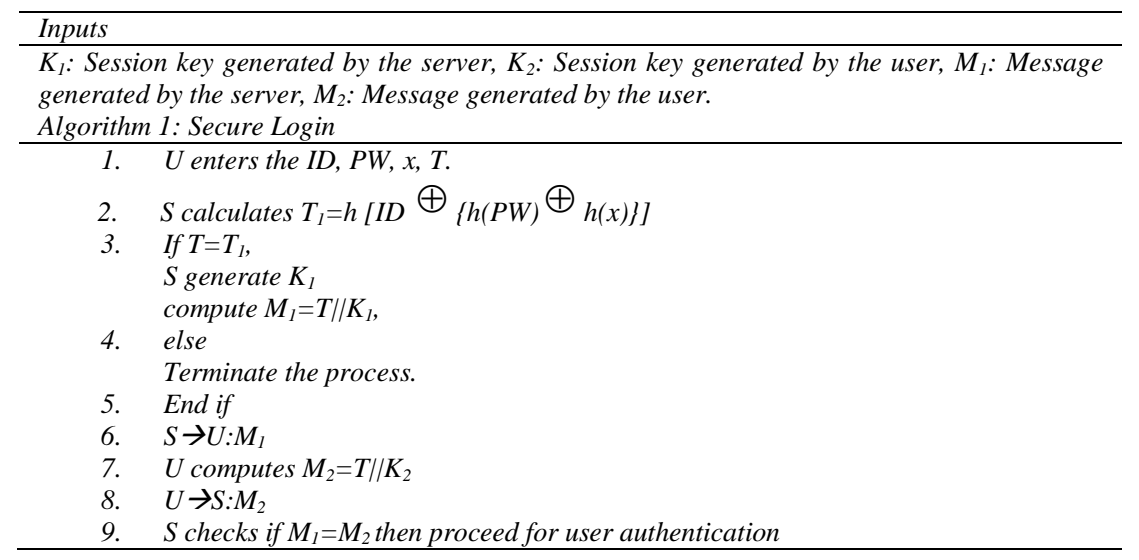

Finally, the user authentication process performed which is used for confirming the identity of the user which can be done by the server in the cloud. The algorithm 2 shows the steps involved in the process of user authentication.

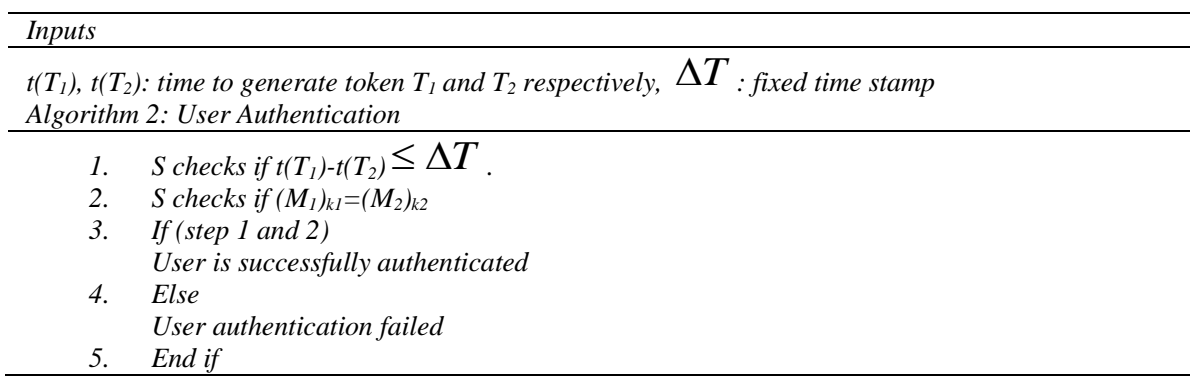

\section{REAL TIME CONTROL AND MONITORING (RTCM) DEVICE}

This section presents the steps that conducted during the experimental study of proposed architecture before discussing the results achieved. Figure 4 is showing the single line diagram (Ref. to Figure 1 above) on HMI screen of real-time control and monitoring device for grid power control.

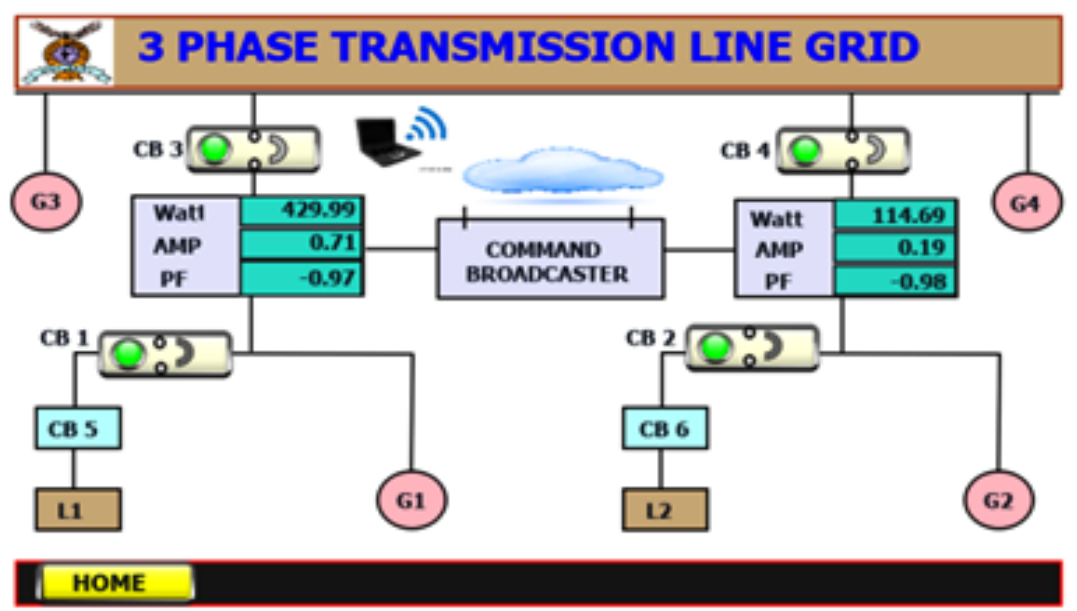

Figure 4. Single line diagram on HMI screen of RTCM device 
Figure 4 demonstrated the grid which is parallel connected with four generators. The G3 and G4 represent two generators of the electric grid to protect against the over-drawl conditions. Generators G1 and G2 represent two regions of micro-grid that the need to be protected against the over-drawl conditions. The L1 and L2 are the local loads of generators G1 and G2 while 3 phase transmission line grids are considered as grid loads to be isolated in over-drawling conditions. Figure 5 demonstrates the actual hardware panel of the RTCM device. This is the fabricated hardware model of the RTCM device. Figure 6 shows the labeled diagram for the same fabricated hardware model designed in this paper. Figure 6 helps to understand the exact design used to for the hardware panel in this work.

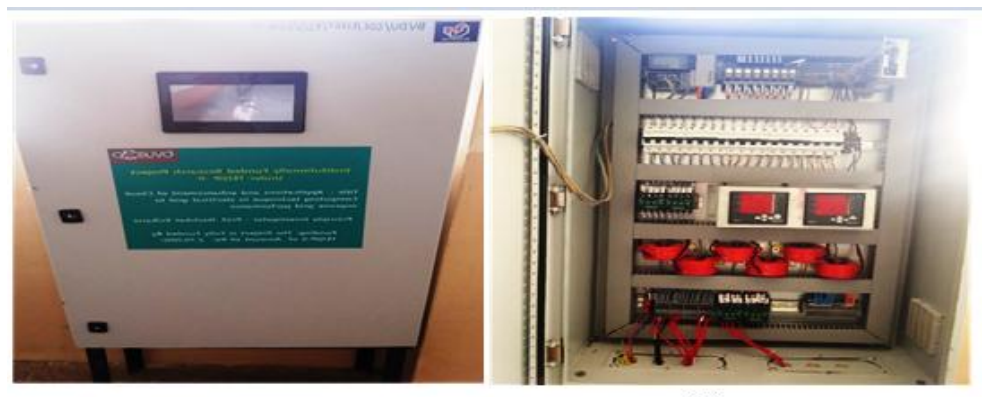

(a) (b)

Figure 5. Fabricated hardware model of RTCM device (a) Front interface and (b) Load generators

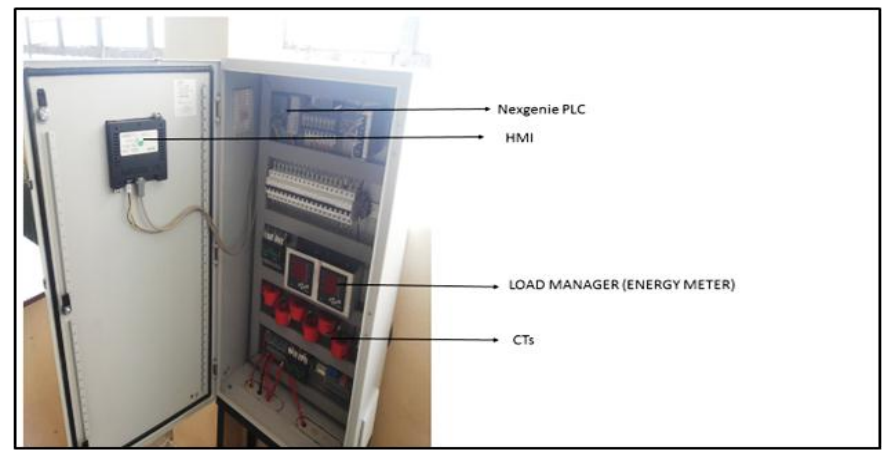

Figure 6. Labeled diagram for proposed hardware panel of RTCM Device

\section{EXPERIMENTAL RESULTS}

As per design and settings discussed in this Section 3 and 4 of this paper, the experimental results demonstrated in this section. The proposed model was implemented and tested using HMI which uses EXOR E Smart (7-inch Medium) device and J Mobile Software. In addition to that, the power flow in the grid is continuously monitored using PLC and Energy Meter. Figure 7 is showing the home screen displayed at HMI of RTCM device.

There are three tabs given on HMI screen in order fetch the current single line diagram (SLD) for remote installed grid power systems. Parameters tab is used to monitor the grid power system using various parameters of electrical data. Finally, the settings tab is used to change the settings.

First, we present the parameters monitoring results. After the complete setup and design of the realtime model for monitoring and control of grid power system remotely using cloud computing, there is parameter monitoring interface provided on HMI. Using this parameter monitoring interface, we can monitor the instantaneous values of various elements like a Active power, Reactive Power, phase voltages, phase currents, the Apparent power of loads of Grid 1 and Grid 2. After clicking on SLD tab, we can get the result for 3 phase transmission line grid as shown in Figure 8.

In this work, we performed the generation and transmission based on 3-stage only. The optional transmission is likewise 3-stage through the circulation to a definitive client might be 3-stage or single-stage contingent on the necessities of the clients. Finally, the setting tab elaborated in below Figure 10 and Figure 11. Initially, it is necessary to set the parameter values of the grid. The working of the network 
depends upon the initial parameter values which have been set by the user. Figure 10 shows the initial parameter values of grid 1 and grid 2. Then we can monitor the actual value of the load and thus can vary the set reference point as per our requirement in both the grids Grid 1 and Grid 2. We can also change the delay of operation of circuit breakers from this screen. Figure 9 is showing the values of parameters after the cloud interface which is changing from the initial set values.

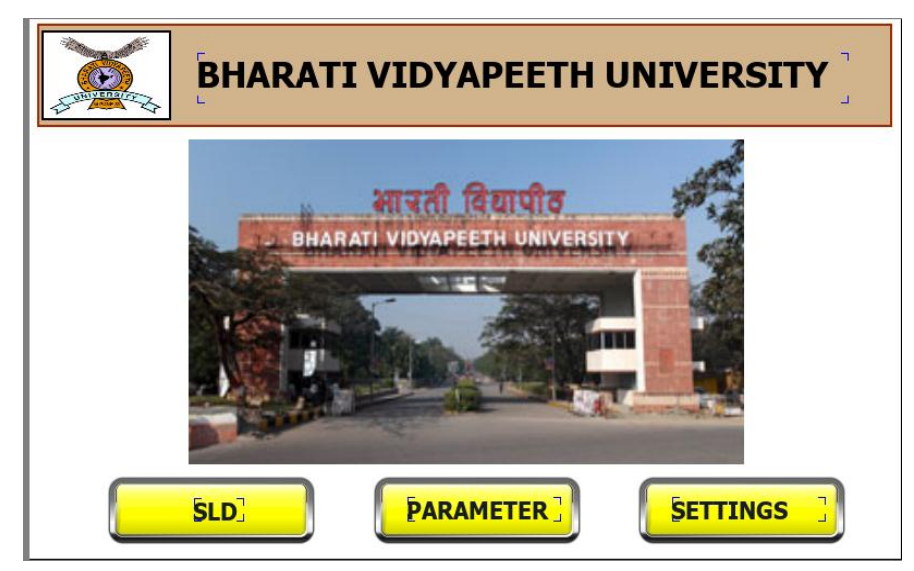

Figure 7. Home screen of touch on HMI of RTCM device

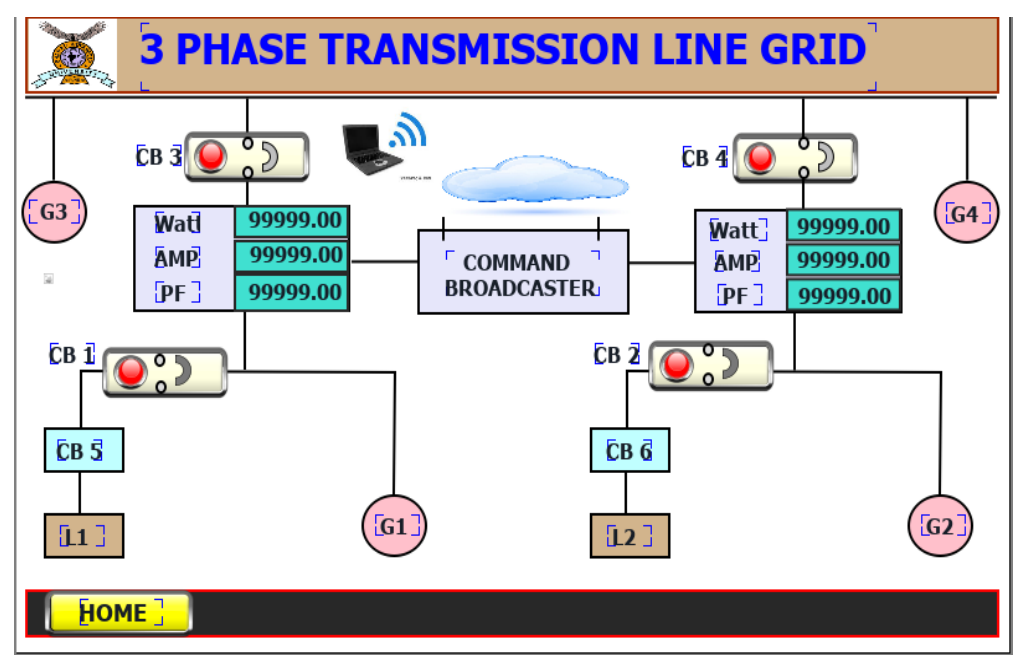

Figure 8. SLD diagram of the Transmission network on HMI
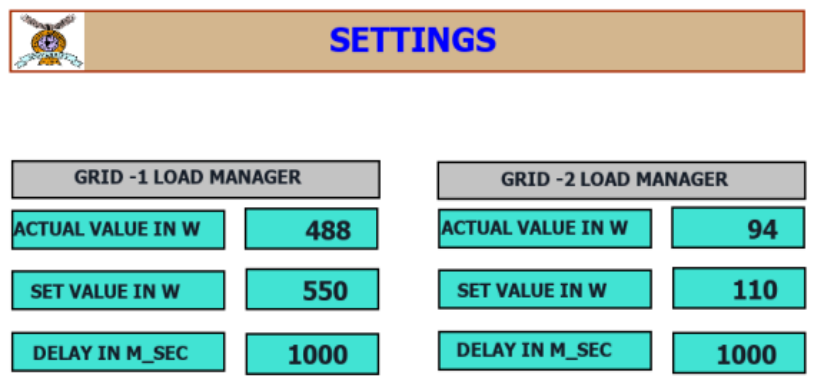

HOME

Figure 9. Initial Setting of two grids through the HMI 


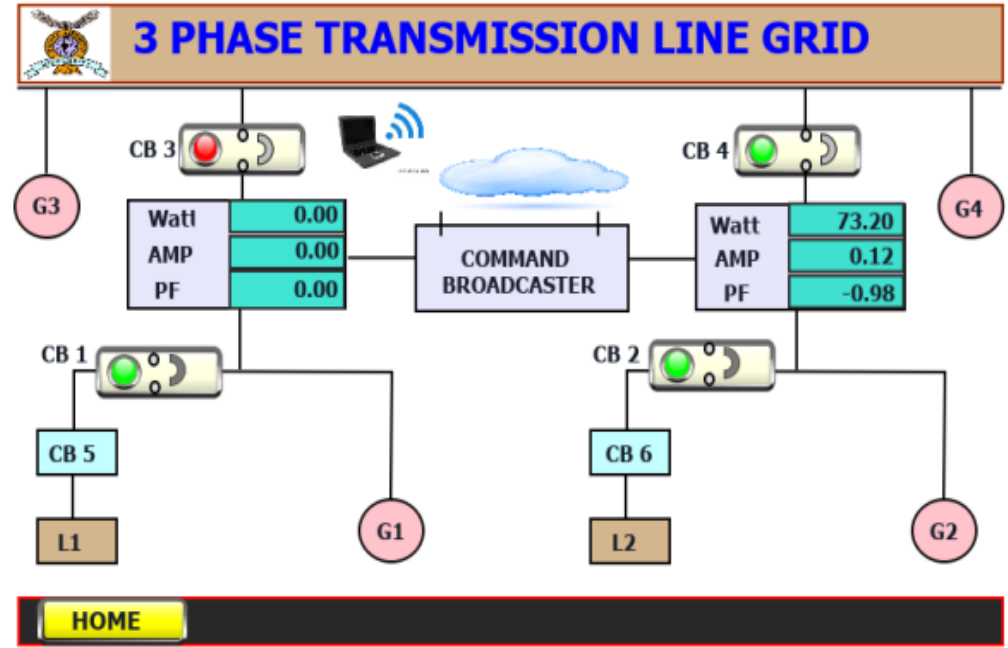

Figure 10. Operation of Circuit Breaker 3 by PLC

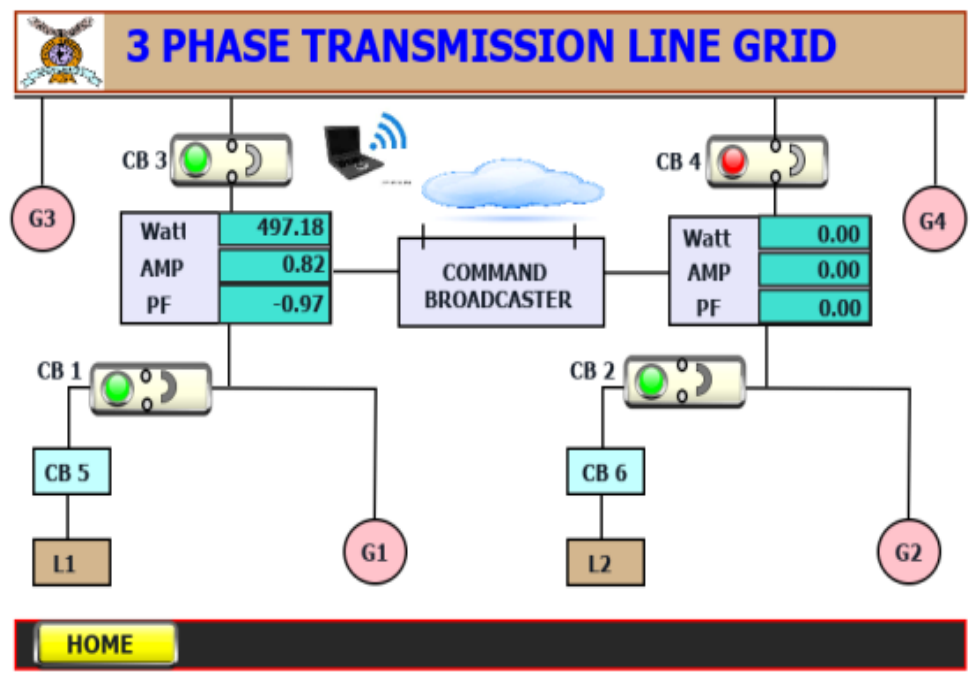

Figure 11. Operation of Circuit Breaker 4 by PLC

\section{CONCLUSION AND FUTURE WORK}

In this paper, we focused on benefits of using the cloud framework and ICT standards for the smart grid. The smart grid is composed of smart measuring of electrical devices from the data which is collected and stored at the cloud server for the further remote monitoring process. We presented the real-time design for the control and monitoring of grid system electrical data remotely using the cloud computing frameworks. We designed the hardware panel which is used to monitor the system parameters continuously and enable the authorized users to access, control and monitor the electrical data through the user authentication process. We believe that helps in continuously monitoring the grid parameters and help to detect the grid failure at the earliest and by verifying the user authentication, the user can control or prevent grid failure from anywhere in the world. In this paper, our initial step towards the future smart grid presented, for future work, it will be interesting to work on designing efficient energy management methods and optimal power delivery.

\section{REFERENCES}

[1] "Cloud Computing Applications for Smart Grid: A Survey" -Samaresh Bera, Sudip Misra,Senior Member, IEEE, Joel J. P. C. Rodrigues, Senior Member, IEEE.

[2] J. Popeanga, "Cloud Computing and Smart Grids," Database system journal, vol. 3, no. 3, pp. 57-66,2012. 
[3] QDR Q 2006 Benefits of demand response in electricity markets and recommendations for achieving them (USA: U.S. Department of Energy) Chiu, Ipakchi A, Chuang A, Qiu B, Brooks D, Koch E, Zhou J, Zientara M, Precht P and Burke R 2009 Framework for integrated demand response (DR) and distributed energy resources (DER) models NAESB \& UCAIug.

[4] Siano P 2014 Demand response and smart grids - a survey Renewable and Sustainable Energy Reviews 30 461-78

[5] Kim H, Kim Y J, Yang K and Thottan M 2011 Cloud-based demand response for smart grid: architecture and distributed algorithms Proc. Int. Conf. on Smart Grid Communications (SmartGridComm) (Brussels) (USA: IEEE) 398-403.

[6] Soultanis N L, Papathanasiou S A and Hatziargyriou N D, 2007, "A stability algorithm for the dynamic analysis of inverter dominated unbalanced LV microgrids," IEEE Transactions on Power Systems 22 294-304.

[7] Chen M, Bai X Zhu Y and Wei H, 2012, "Research on power dispatching automation system based on cloud computing," Proc. Innovative Smart Grid Technologies-Asia (ISGT Asia) (Tianjin) (USA: IEEE) 1-6.

[8] Liang Z, Lei Z, Min-hui G and Xiao-liang B, 2011, "Research and application on the cloudcomputing-based power dispatching IT architecture," Proc. Power and Energy Engineering Conference (APPEEC) Asia-Pacific (Wuhan) (USA: IEEE) 1-4.

[9] Yang C T, Chen W S, Huang K L, Liu J C, Hsu W H and Hsu C H, 2012, "Implementation of smart power management and service system on cloud computing," Proc. 9th Int. Conf. on Ubiquitous Intelligence \& Computing and Autonomic \& Trusted Computing (UIC/ATC) (Fukuoka) (USA: IEEE) 924-9.

[10] Rajeev T and Ashok S, 2011, "A cloud computing approach for power management of microgrids," Proc. Innovative Smart Grid Technologies-India (ISGT India) (Kollam, Kerala) (USA: IEEE) 49-52.

[11] Vázquez, Constantino, Eduardo Huedo, Rubén S. Montero, and Ignacio M. Llorente, "On the use of clouds for grid resource provisioning," Future Generation Computer Systems Vol. 27, no. 5, pp. 600-605, 2011.

[12] Moslehi, Khosrow, and Ranjit Kumar, "A reliability perspective of the smart grid," IEEE Transactions on Smart Grid Vol.1, no. 1, pp. 57-64, 2010.

[13] Fang, Baling, Xiang Yin, Yi Tan, Canbing Li, Yunpeng Gao, Yijia Cao, and Jianliang Li, "The contributions of cloud technologies to smart grid," Renewable and Sustainable Energy Reviews Vol.59, pp. 1326-1331, 2016.

[14] Fang, Xi, Dejun Yang, and Guoliang Xue, "Evolving smart grid information management cloudward: A cloud optimization perspective," IEEE Transactions on Smart Grid Vol.4, no. 1, pp. 111-119, 2013.

[15] Mehmi, Sandeep, Harsh K. Verma, and A. L. Sangal, "Simulation modeling of cloud computing for smart grid using CloudSim,” Journal of Electrical Systems and Information Technology Vol.4, no. 1, pp. 159-172, 2017.

\section{BIOGRAPHIES OF AUTHORS}
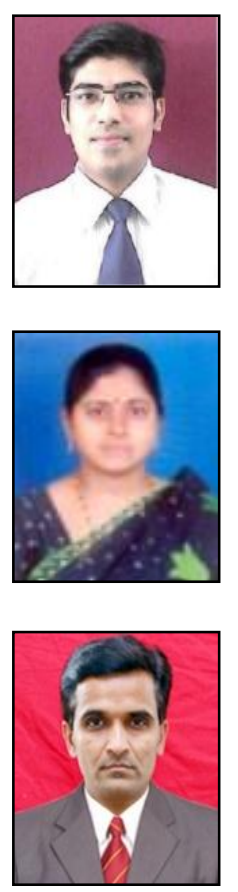

Nachiket Kulkarni is presently pursuing his Ph.D. from Koneru Lakshmaiah Education Foundation, Vaddeswaram, Guntur, Andhra Pradesh, India - $522502 \mathrm{He}$ is working as an Assistant Professor in Electrical Engineering Department, Bharati Vidyapeeth Deemed to be University College of Engineering, Pune. His research interests are Power Quality and Cloud Computing applications in smart grid.

S. V. N. L. Lalitha obtained her Ph.D from National Institute of Technology, Warangal, India. She is working as a Professor in Electrical \& Electronics Engineering Department, Koneru Lakshmaiah Education Foundation, Vaddeswaram, Guntur, Andhra Pradesh, India - 522502. Her research interests are transmission pricing in restructured power systems, distribution system reconfiguration, smart grids and Meta Heuristic Techniques applied to power system operation and control.

Sanjay A. Deokar obtained his Ph.D from SGGS Nanded, Maharashtra, India. He is working as a Professor in Electrical Engineering Department, ZES's Zeal College of Engineering and Research, Narhe, Pune. His research interests are Power Quality, Renewable Energy and Smart Grid. 